\title{
Carcinoembryonic antigen levels of tumor-draining venous blood as a prognostic marker in colon cancer
}

Sun Hyung Choi', Seung Yoon Yang ${ }^{1}$, Yoon Dae Han ${ }^{1}$, Min Soo Cho' ${ }^{1}$ Hyuk Hur ${ }^{1}$, Kang Young Lee', Nam Kyu Kim', Byung Soh Min ${ }^{1,2}$ 'Department of Surgery, Severance Hospital, Yonsei University College of Medicine, Seoul;

${ }^{2}$ Open NBI Convergence Technology Research Laboratory, Severance Hospital, Yonsei Cancer Center, Seoul, Korea

Purpose: Carcinoembryonic antigen (CEA) is a tumor marker for colorectal cancer (CRC) related to recurrence and prognosis. We examined the ability of the CEA level measured directly from a tumor drainage vein (dCEA) to predict the prognosis of CRC more accurately than those from a peripheral vein (pCEA).

Methods: Fifty-two patients who received curative resection for colon adenocarcinoma were enrolled. The patients were categorized into two groups according to normal pCEA $(<5.9425 \mathrm{ng} / \mathrm{mL}, \mathrm{n}=24)$ or elevated pCEA levels ( $\geq 5.9425 \mathrm{ng} / \mathrm{mL}, \mathrm{n}=28)$. Blood was sampled at the time of surgery simultaneously from the tumor drainage vein and from the peripheral vein.

Results: The clinicopathologic variables showed no significant difference between the two groups. Patients with dCEA levels $<20.192 \mathrm{ng} / \mathrm{mL}$ showed better disease-free $(P=0.009)$ and overall survival $(P=0.033)$ curves than those with dCEA levels $\geq 20.192 \mathrm{ng} / \mathrm{mL}$. Elevated dCEA levels were a significant prognostic factor for overall survival and disease-free survival in Cox proportional hazard model analysis (hazard ratio $[\mathrm{HR}]=399 ; 95 \%$ confidence interval $[\mathrm{Cl}], 16.4-9,747 ; \mathrm{P}<0.001 ; \mathrm{HR}=9.39,95 \% \mathrm{Cl}, 1.29-68.006 ; \mathrm{P}=0.026)$. In subgroup analysis, we compared the data of normal range of dCEA group and elevated dCEA group with normal pCEA; the overall survival rate of patients with normal dCEA was better and the disease-free survival rate was significantly better $(P=0.003)$.

Conclusion: CEA levels from a tumor drainage vein can be used as more accurate prognostic markers than levels from a peripheral vein in patients with colon cancer.

Keywords: Carcinoembryonic antigen, Prognosis, Colorectal cancer

\section{INTRODUCTION}

Carcinoembryonic antigen (CEA) is one of the most extensively used clinical tumor markers in cases of colorectal cancer (CRC). The primary use of serum CEA levels as a tumor marker is in post-surgical surveillance of CRC to detect distant metastasis or tumor recurrence [1]. Serial CEA measurements can detect recurrent CRC with a sensitivity of between $60 \%$ and $95 \%$, and a speci-

Received: Aug 6, 2017 Accepted: Dec 13, 2017

Correspondence to: Seung Yoon Yang

Department of Surgery, Severance Hospital, Yonsei University College of

Medicine, 50 Yonsei-ro, Seodaemun-gu, Seoul 03722, Korea

Tel: +82-10-8552-9396, Fax: +82-2-313-8289

E-mail: syyy2000@yuhs.ac

Copyright @ Corean Society of Surgical Oncology

This is an Open Access article distributed under the terms of the Creative Commons Attribution Non-Commercial License (http://creativecommons.org/licenses/by-nc/4.0) which permits unrestricted non-commercial use, distribution, and reproduction in any medium, provided the original work is properly cited. ficity of between $34 \%$ and $91 \%$ [2,3]. Thus, monitoring CEA levels after treatment provides an early indication and allows for secondary surgery or other treatment modalities. In addition, serum CEA levels can be used as prognostic indicators. A high preoperative CEA level in CRC is associated with poor 5-year overall survival, while a low preoperative level is associated with good survival $[4,5]$.

However, there are limitations (false positive and false negative test results) to the use of serum CEA as a tumor marker. Elevated serum CEA is detected in cigarette smokers, patients with benign neoplasm, and in $15 \%$ to $20 \%$ of subjects with inflammatory disorders such as ulcerative colitis, Crohn's disease, pancreatitis, liver disease, and pulmonary infections. Thus, elevated plasma CEA levels are not specific for cancer, although very high concentrations (> $20 \mathrm{ng} / \mathrm{mL}$ ) are highly suggestive of malignancy [6,7]. In case of a false negative result, serum CEA level varies by tumor grade, liver status, and the ploidy status of the tumor. For example, the mean CEA concentration in a poorly differentiated colorectal neoplasm 
is lower than in a well-differentiated or moderately differentiated tumor, and the CEA level of tumors with a near-diploid pattern is lower than in tumors with aneuploidy CRC [2]. These examples indicate limitations of the use of CEA as a prognostic indicator.

In colon cancer, the bloodstream first carries the cancer cells to the superior/inferior mesenteric veins (SMV/IMV), the portal vein, and then the liver, where the vessels divide into capillaries. Thus, for colorectal cancers, the SMV/IMV and portal vein act as drainage veins (DVs). Previous studies showed that the elevation of CEA level was correlated with Dukes' staging, lymphovascular invasion, and infiltration of cancer cells in immunohistochemical staining analysis, and that the DV blood CEA (dCEA) level was more elevated than the peripheral CEA (pCEA) level $[8,9]$. These findings suggest that the route of CEA transfer from the tumor to the peripheral blood could be an important contributing factor to the control of peripheral CEA level, and that CEA from a DV might be more sensitive to histopathologic tumor characteristics.

On the basis of previous studies, we can hypothesize that the CEA level from the DV (SMV or IMV) could more accurately reflect histopathologic tumor characteristics than from the peripheral vein (PV) because the CEA is cleared from circulation by the liver, and that the CEA from the DV might be more strongly correlated with patients' oncologic outcomes. The aim of this study was to explore whether the CEA level measured directly from the tumor DV can predict prognosis more accurately than that from the PV by increasing the sensitivity of CEA detection.

\section{METHODS}

\section{Ethics statement}

This investigation has been conducted in accordance with the ethical standards, the Declaration of Helsinki, and national and international guidelines. The present study was approved by the Institutional Review Board of the Severance Hospital (IRB no. 4-20170157).

\section{Patients}

A total of 52 patients who received curative resection between 2004 and 2005 at Yonsei University Severance Hospital, Seoul, Korea for pathologically confirmed adenocarcinoma originating in the colon were enrolled. Patients who had metastasis, palliative resection, previous cancer treatment, or no available paired blood samples from both the tumor DV and the PV were excluded.

\section{Blood samples and ELISA}

Blood samples $(5 \mathrm{~mL})$ were drawn from the PV and tumor DV and placed in plain tubes. PV blood was obtained 1 hour prior to surgical incision. DV blood was obtained prior to the ligation of any branch of the SMV in patients with proximal colon cancer and prior to ligation of the IMV in patients with distal colon cancers. Samples were immediately centrifuged, and plasma and serum were separately stored at $-70^{\circ} \mathrm{C}$ until analysis. enzyme-linked immunosorbent assay (ELISA) was performed using commercially available kits (IBL-Hamburg GmbH, Hamburg, Germany) according to manufacturer's instructions. Diluted serum was transferred to wells of plates pre-coated with primary antibody. Following the recommended incubation period, plates were washed with buffer solution, and substrate solution was added and incubated as instructed. The wells were developed with a color-reagent. Stop solution was added to each well after incubation and the optical density was measured at a wavelength of $450 \mathrm{~nm}$ using automated optical densitometry. Each sample was run in triplicate, and the mean value was used for analysis. If the $\mathrm{R}^{2}$ values of the standard solutions was $<0.98$, data from the plate were excluded.

\section{Statistical analysis}

Statistical analysis was performed by using SPSS ver. 18.0 (SPSS Inc., Chicago, IL, USA).

Analyzed clinical parameters included age, gender, tumor node metastasis (TNM) stage, histological grade, and lymphovascular invasion. Data on molecular factor levels are shown as the mean \pm standard deviation. The Mann-Whitney U test, Fisher's exact test, and McNemar test were used to compare differences in molecular factor levels between the clinical and pathological features. A Cox proportional hazards analysis was used to evaluate the relationship between pathological features, plasma molecular factor levels, and overall survival. A P-value $<0.05$ was considered significant.

\section{RESULTS}

\section{Patient characteristics}

A total of 52 patients were enrolled in this study. The mean age was 60 years; approximately half of patients had stage 3 disease (Table 1). The mean pCEA and dCEA levels were $14.4 \pm 32.0 \mathrm{ng} / \mathrm{mL}$ and $65.2 \pm 144.7 \mathrm{ng} / \mathrm{mL}(\mathrm{P}=0.001)$, respectively. There was a moderate correlation between pCEA and dCEA levels (Pearson correlation coefficient: 0.6736).

Patients were then divided into 2 groups according to whether their peripheral CEA exceeded the reference value of $5.9425 \mathrm{ng} /$ $\mathrm{mL}$ (Fig. 1A). Clinicopathologic variables were compared; none was significantly different (Table 1). The dCEA levels in each group were $31.8 \pm 31.9 \mathrm{ng} / \mathrm{mL}$ and $93.8 \pm 192.0 \mathrm{ng} / \mathrm{mL}$, respectively, but were not statistically different $(\mathrm{P}=0.103)$. 
Table 1. Patient characteristics

\begin{tabular}{|c|c|c|c|c|}
\hline Variable & Total & pCEA $<5 \mathrm{ng} / \mathrm{mL}$ & pCEA $\geq 5 \mathrm{ng} / \mathrm{mL}$ & P-value \\
\hline Age (yr) & $59.9 \pm 9.4$ & $60.0 \pm 10.6$ & $59.8 \pm 8.4$ & 0.946 \\
\hline Sex & & & & 0.948 \\
\hline Male & 29 & 14 & 15 & \\
\hline Female & 23 & 10 & 13 & \\
\hline Blood transfusion & 6 & 3 & 3 & 1 \\
\hline \multicolumn{5}{|l|}{ Surgical margin involvement } \\
\hline Proximal & 0 & 0 & 0 & Not significant \\
\hline Distal & 0 & 0 & 0 & Not significant \\
\hline Clavien-Dindo grade of complications & & & & 0.53 \\
\hline 2 & 18 & 6 & 12 & \\
\hline 3 & 7 & 3 & 4 & \\
\hline 4 & 2 & 1 & 1 & \\
\hline TNM stage & & & & 0.542 \\
\hline I & 5 & 2 & 3 & \\
\hline$\|$ & 15 & 6 & 9 & \\
\hline III & 25 & 14 & 11 & \\
\hline IV & 7 & 2 & 5 & \\
\hline No. of positive lymph nodes & 3.4 & 3.5 & 3.4 & 0.892 \\
\hline No. of harvested lymph nodes & 21.7 & 20.7 & 22.5 & 0.534 \\
\hline Histologic grade (differentiation) & & & & 0.519 \\
\hline Well & 8 & 4 & 4 & \\
\hline Moderate & 33 & 15 & 18 & \\
\hline Poor & 7 & 2 & 5 & \\
\hline Mucinous & 4 & 3 & 1 & \\
\hline Vascular invasion & 21 & 8 & 13 & 0.499 \\
\hline Lymphatic invasion & 21 & 12 & 9 & 0.305 \\
\hline Tumor size $(\mathrm{cm})$ & 4.6 & 5.3 & 4.0 & 0.061 \\
\hline Body mass index $\left(\mathrm{kg} / \mathrm{m}^{2}\right)$ & 23.0 & 22.9 & 23.1 & 0.782 \\
\hline Previous abdominal surgery & 10 & 6 & 4 & 0.532 \\
\hline Tumor location & & & & 0.256 \\
\hline Right & 9 & 5 & 4 & \\
\hline Left & 43 & 19 & 24 & \\
\hline Adjuvant chemotherapy & 44 & 20 & 24 & $>0.99$ \\
\hline pCEA level (ng/mL) & 14.4 & 1.6 & 25.4 & 0.005 \\
\hline dCEA level (ng/mL) & 65.2 & 31.8 & 93.8 & 0.103 \\
\hline
\end{tabular}

Values are presented as mean \pm standard deviation or number (\%).

PCEA, peripheral vein CEA; CEA, carcinoembryonic antigen; TNM, tumor node metastasis; dCEA, drainage vein CEA.

\section{Oncologic outcomes and CEA levels}

To determine cut-off values for pCEA and dCEA levels, receiver operating characteristic (ROC) curves were analyzed. Through ROC curve analysis, a point most correlated to recurrence with the highest sensitivity and specificity was calculated for both pCEA and dCEA levels. For pCEA, it was determined to be $5.9425 \mathrm{ng} /$ $\mathrm{mL}$, the same as the reference value, for which sensitivity and specificity were 0.667 and 0.588 , respectively (Fig. 1A). For dCEA, the point was $20.192 \mathrm{ng} / \mathrm{mL}$; sensitivity and specificity were 0.833 and
0.529, respectively (Fig. 1B).

For given cut-off values of pCEA and dCEA, Kaplan-Meier survival analyses with $\log$ rank tests were performed. When the analysis was performed according to a pCEA level below $5.9425 \mathrm{ng} / \mathrm{mL}$, both disease-free and overall survival curves were not significantly different (Fig. 2). However, patients with dCEA below $20.192 \mathrm{ng} /$ $\mathrm{mL}$ showed better disease-free $(\mathrm{P}=0.009)$ and overall survival $(\mathrm{P}=0.033$ ) curves than those with dCEA $\geq 20.192 \mathrm{ng} / \mathrm{mL}$ (Fig. 3).

The Cox proportional hazard model analysis revealed the dCEA 
ROC curves of peripheral vein CEA

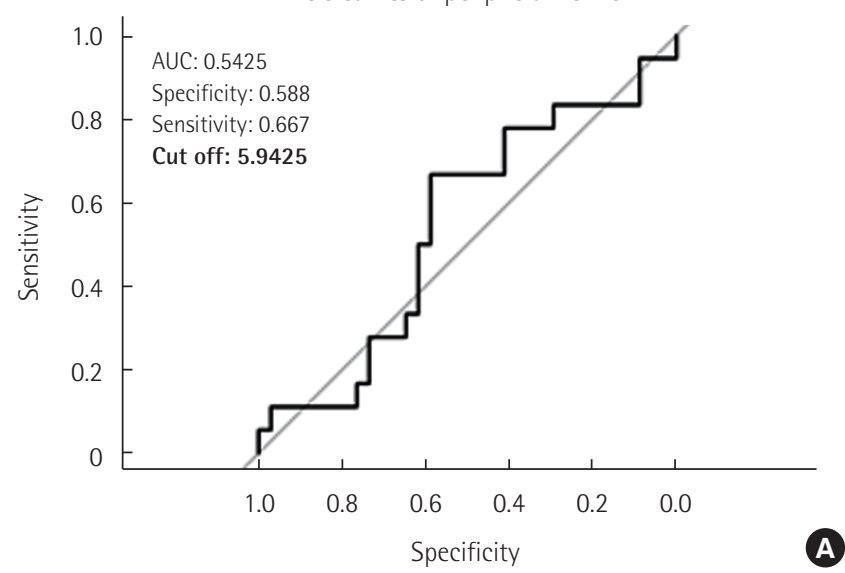

ROC curves of drainage vein CEA

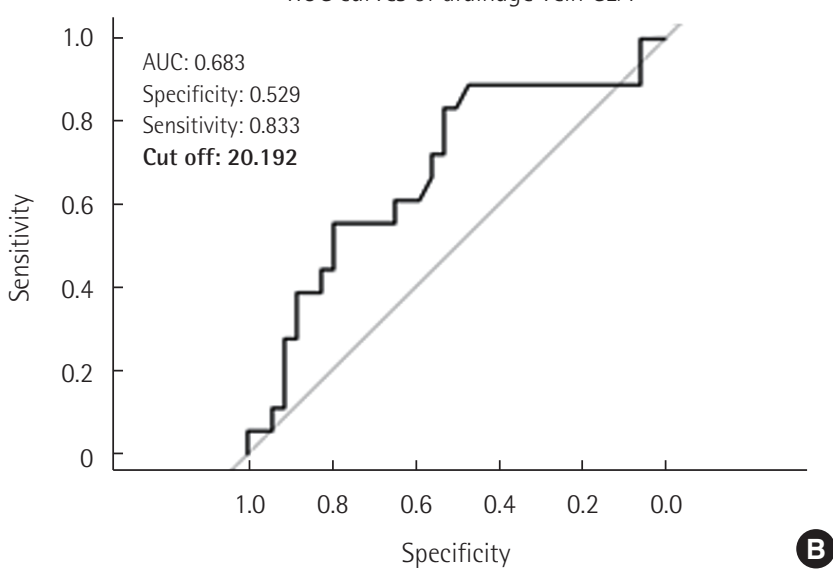

Fig. 1. Receiver operating characteristic curve analysis pCEA (A) and dCEA (B). AUC, area under the curve; CEA, carcinoembryonic antigen; pCEA, peripheral vein CEA; dCEA, drainage vein CEA.
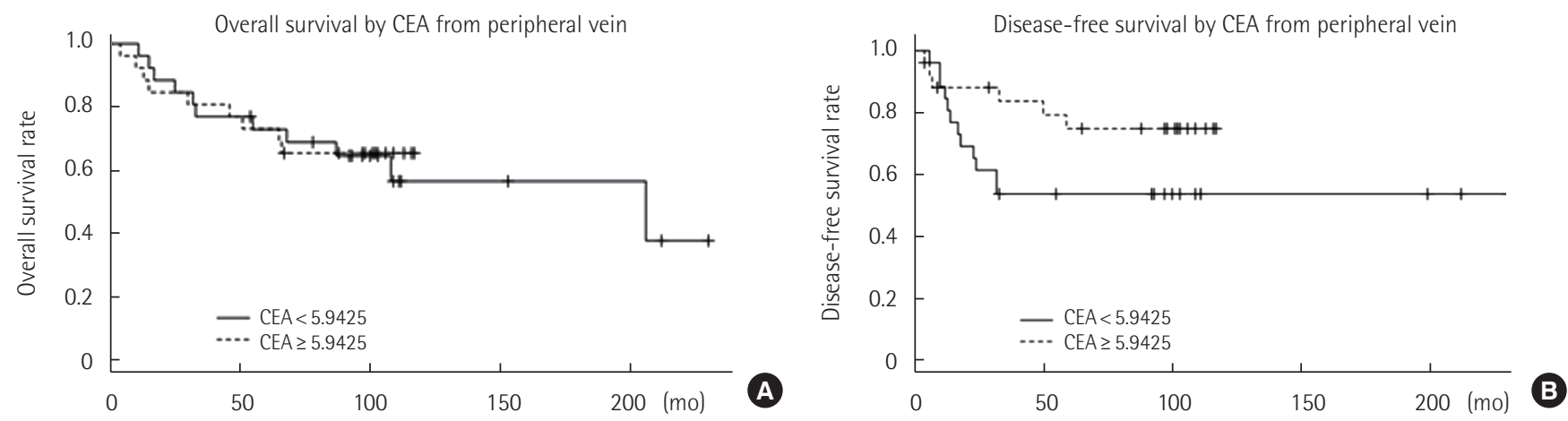

Fig. 2. Disease-free (A) and overall survival (B) curves according to pCEA level. CEA, carcinoembryonic antigen; pCEA, peripheral vein CEA.
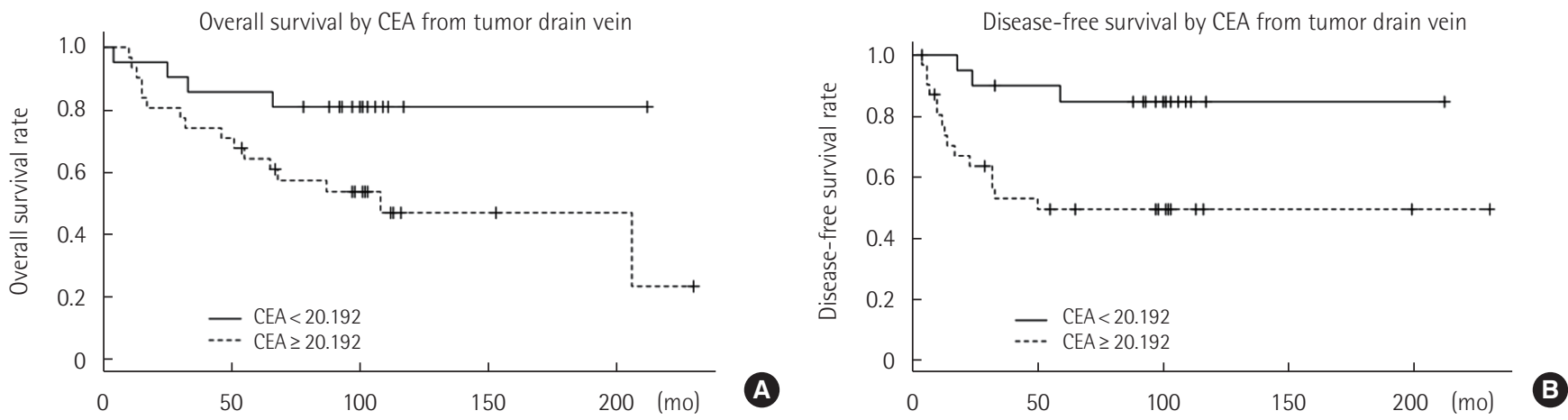

Fig. 3. Disease-free $(A)$ and overall survival $(B)$ curves according to dCEA level. CEA, carcinoembryonic antigen; dCEA, drainage vein CEA.

level to be a significant prognostic factor for disease-free (hazard ratio $[\mathrm{HR}]=399 ; 95 \%$ confidence interval $[\mathrm{CI}], 16.4-9,747 ; \mathrm{P}<0.001)$ and overall survival $(\mathrm{HR}=9.39 ; 95 \% \mathrm{CI}, 1.29-68.006 ; \mathrm{P}=0.026)$ (Table 2). For disease-free survival, significant poor prognostic factors other than high dCEA level included a poorly differentiated tumor, complications, a Clavien-Dindo grade $\geq 3$, patients $>60$ years of age, TNM stage 3 or 4 , and intraoperative blood transfu- sion. For overall survival, factors included poorly differentiated tumors, complications, a Clavien-Dindo grade $\geq 3$, or a lymph node harvest below 12 .

Significance of dCEA levels in patients with normal pCEA A subgroup analysis was performed to determine whether the dCEA level has prognostic significance in patients with a normal 
Table 2. Cox proportional hazard model for prognostic factor analysis

\begin{tabular}{|c|c|c|c|c|c|c|}
\hline \multirow[b]{2}{*}{ Variable } & \multicolumn{3}{|c|}{ Overall survival } & \multicolumn{3}{|c|}{ Disease-free survival } \\
\hline & Hazard ratio & $\begin{array}{c}95 \% \text { Confidence } \\
\text { intervals }\end{array}$ & P-value & Hazard ratio & $\begin{array}{l}\text { 95\% Confidence } \\
\text { interval }\end{array}$ & P-value \\
\hline Poorly differentiated & & & & 69.4 & $3.3-1,476.8$ & 0.006 \\
\hline Complication (Clavien-Dindo grade) & 23.8 & $1.4-408.3$ & 0.028 & 15.6 & $1.4-177.8$ & 0.027 \\
\hline No. of harvested lymph nodes $<12$ & & & & $2,121.8$ & $4.6-979,502.4$ & 0.015 \\
\hline Age $>60 \mathrm{yr}$ & 7.8 & $1.7-36.5$ & 0.010 & & & \\
\hline TNM stage $\geq 3$ & $1,394.1$ & $1.0-1,854,637.8$ & 0.049 & & & \\
\hline Intraoperative blood transfusion & 34.1 & $3.1-380.2$ & 0.007 & & & \\
\hline $\mathrm{dCEA} \geq 20.192 \mathrm{ng} / \mathrm{mL}$ & 9.4 & $1.3-68.0$ & 0.026 & 399.4 & $16.4-9,747.8$ & $<0.001$ \\
\hline
\end{tabular}

TNM, tumor node metastasis; dCEA, drainage vein carcinoembryonic antigen.
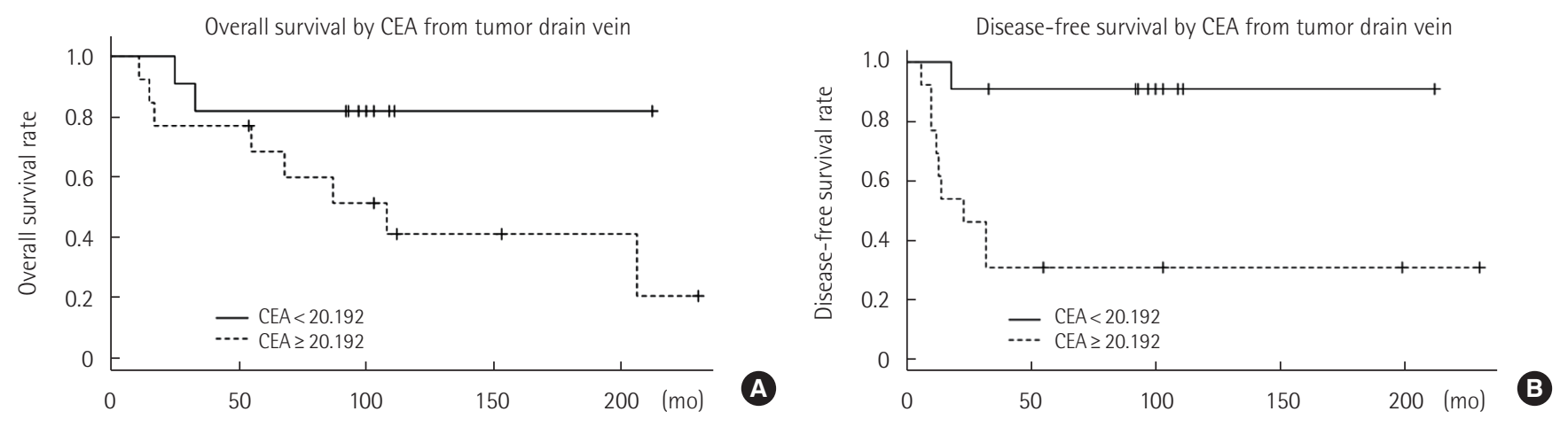

Fig. 4. Disease-free $(A)$ and overall survival $(B)$ curves according to dCEA level in the patients with a pCEA level in the normal range. CEA, carcinoembryonic antigen; dCEA, drainage vein CEA; pCEA, peripheral vein CEA.

pCEA level. Of 51 patients in this study, $24(47.06 \%)$ had a pCEA level in the normal range of $<5.9425 \mathrm{ng} / \mathrm{mL}$; these patients were divided into 2 groups according to whether their dCEA level was below the calculated cut-off value $(20.192 \mathrm{ng} / \mathrm{mL})$. Kaplan-Meier survival analysis revealed that patients with a dCEA level $\geq 20.192$ $\mathrm{ng} / \mathrm{mL}$ showed significantly poorer disease-free survival than those with dCEA $<20.192 \mathrm{ng} / \mathrm{mL}(\mathrm{P}=0.009)$ (Fig. 4). A Cox proportional hazard model revealed that none of the analyzed variables, including the dCEA level, were significant prognostic factors for both disease-free and overall survival.

\section{DISCUSSION}

CEA is a tumor marker generally used as an early indicator of tumor recurrence and prognosis for CRC [10,11]. Consistent elevation of serum CEA levels is a concerning sign of disease recurrence, and correlates sufficiently with CRC activity. For that reason, routine measurement of preoperative CEA levels before colorectal surgery for CRC patients is primarily suggested for subsequent postoperative surveillance by the Guidelines of the National Comprehensive Cancer Network (NCCN) and the European Society of
Medical Oncology (ESMO) [1,12,13].

However, the significance of preoperative CEA levels as a prognostic factor for recurrence has clear limits [14-16]. Serum CEA has relatively low sensitivity, which may be attributed in part to nonCEA producing tumors that produce false negative results [3]. It is also well known that low-level elevation of CEA, between 5 and 10 $\mathrm{ng} / \mathrm{mL}$, has a substantial likelihood of representing false-positive elevation; Litvak et al. found that the $93 \%$ of isolated, single-occurrence elevations of CEA level with spontaneous normalization were low-level elevations of between 5.1 and $10.0 \mathrm{ng} / \mathrm{mL}$ [7].

The pCEA level is influenced by various pathophysiologic conditions including production of cancer cells [17], excretion of CEA into surrounding tissues, and entry into the lymphatic system and blood stream $[18,19]$. In that sense, measuring dCEA is considered to be more likely to reflect the status of the tumor $[17,19]$. The ability of the level of dCEA to predict CRC prognosis has not yet been established and is not clinically used. Therefore, in this study, we have attempted to evaluate the possibility of dCEA levels as prognostic markers in CRC compared with pCEA levels.

There was no significant difference in the overall survival rate and disease-free survival rate between patients with normal pCEA 
and increased pCEA values. However, both the overall survival rate $(\mathrm{P}=0.033)$ and the disease-free survival rate $(\mathrm{P}=0.009)$ of the group with normal dCEA levels were significantly better than those in the group with increased dCEA levels. Elevated dCEA levels were a significant prognostic factor for overall survival and disease-free survival in the Cox proportional hazard model analysis $(\mathrm{HR}=399 ; 95 \% \mathrm{CI}, 16.4-9,747 ; \mathrm{P}<0.001 ; \mathrm{HR}=9.39 ; 95 \% \mathrm{CI}$, 1.29-68.006; $\mathrm{P}=0.026$ ). These results suggest that dCEA levels may be used to predict prognosis or survival in patients with CRC more accurately than pCEA. The results concur with a previous study of Tabuchi et al. [20] that measurement of dCEA levels is more effective in patients with a poor prognosis than measurement of pCEA levels.

Interestingly, when dCEA was compared in patients with normal pCEA, overall survival rates of patients with normal dCEA was better and disease-free survival rates $(\mathrm{P}=0.003)$ was significantly better than those with elevated dCEA. This result suggests that dCEA levels are more closely related to antigen levels released from cancer cells than pCEA levels, for two reasons. First, CEA is drained from cancer lesions primarily by the hematogenous portal system via the DV, but not by the thoracic duct of the lymphatic system [19]. Second, CEA levels in the PV may be influenced by degradation in the liver [21] or reabsorption in the intestinal wall [22].

Although dCEA may be more useful than pCEA for prediction of the prognosis of CRC, blood collection from a DV is a highly invasive procedure and follow-up collection after surgery is difficult. As serial collection of dCEA is difficult, both dCEA and pCEA are required to predict the prognosis of CRC. There are also concerns about complications of blood collection from a tumor DV. However, during surgery, draining venous blood can be collected without complications, and there were no postoperative complications associated with blood collection from tumor DVs.

Although this study is limited by its retrospective nature and small size, it demonstrated that the determination of dCEA levels can be useful as a predictive and prognostic marker in patients with CRC. The findings of this study increase the subsequent need for further investigations of the usefulness of dCEA as a tumor marker in various clinical settings such as CRC screening, surveillance after curative resection, and monitoring response to chemotherapy in patients with advanced disease.

While concerns exist about serial blood collection from a DV, the current study suggests that dCEA levels can be used as prognostic markers in patients with colon cancer more accurately than pCEA.

\section{CONFLICT OF INTEREST}

No potential conflict of interest relevant to this article was reported.

\section{ACKNOWLEDGMENTS}

This work was supported by a faculty research grant of Yonsei University College of Medicine (6-2015-0160).

\section{REFERENCES}

1. Goldstein MJ, Mitchell EP. Carcinoembryonic antigen in the staging and follow-up of patients with colorectal cancer. Cancer Invest 2005;23:338-51.

2. Duffy MJ. Carcinoembryonic antigen as a marker for colorectal cancer: is it clinically useful? Clin Chem 2001;47:624-30.

3. Tan E, Gouvas N, Nicholls RJ, Ziprin P, Xynos E, Tekkis PP. Diagnostic precision of carcinoembryonic antigen in the detection of recurrence of colorectal cancer. Surg Oncol 2009;18:15-24.

4. Harrison LE, Guillem JG, Paty P, Cohen AM. Preoperative carcinoembryonic antigen predicts outcomes in node-negative colon cancer patients: a multivariate analysis of 572 patients. J Am Coll Surg 1997;185:55-9.

5. Carriquiry LA, Pineyro A. Should carcinoembryonic antigen be used in the management of patients with colorectal cancer? Dis Colon Rectum 1999;42:921-9.

6. Carcinoembryonic antigen: its role as a marker in the management of cancer. Summary of an NIH consensus statement. Br Med J (Clin Res Ed) 1981;282:373-5.

7. Litvak A, Cercek A, Segal N, Reidy-Lagunes D, Stadler ZK, Yaeger $\mathrm{RD}$, et al. False-positive elevations of carcinoembryonic antigen in patients with a history of resected colorectal cancer. J Natl Compr Canc Netw 2014;12:907-13.

8. Tabuchi Y, Deguchi H, Imanishi K, Saitoh Y. Colorectal cancer patients with high risk of hematogenous metastasis: correlation with CEA levels in peripheral and draining venous blood during the period of operation. J Surg Oncol 1991;47:87-91.

9. Talbot RW, Nagorney DM, Pemberton JH, Wieand HS, Ritts RE Jr. Comparison of portal and peripheral blood levels of carcinoembryonic antigen, CA 19-9, and CA 125 tumor-associated antigens in patients with colorectal and pancreatic cancer. Cancer Res 1989; 49:542-3.

10. Gold P, Freedman SO. Demonstration of tumor-specific antigens in human colonic carcinomata by immunological tolerance and absorption techniques. J Exp Med 1965;121:439-62.

11. Becerra AZ, Probst CP, Tejani MA, Aquina CT, Gonzalez MG, Hensley BJ, et al. Evaluating the prognostic role of elevated preoperative carcinoembryonic antigen levels in colon cancer patients: results from the national cancer database. Ann Surg Oncol 2016; 23:1554-61.

12. Benson AB 3rd, Venook AP, Bekaii-Saab T, Chan E, Chen YJ, Coo- 
per HS, et al. Colon cancer, version 3.2014. J Natl Compr Canc Netw 2014;12:1028-59.

13. van de Velde C, Boelens PG, Borras JM, Coebergh JW, Cervantes A, Blomqvist L, et al. EURECCA colorectal: multidisciplinary management: European consensus conference colon \& rectum. Eur J Cancer 2014;50:1.e1-34.

14. Wolmark N, Fisher B, Wieand HS. The prognostic value of the modifications of the Dukes' C class of colorectal cancer: an analysis of the NSABP clinical trials. Ann Surg 1986;203:115-22.

15. Takahashi T, Kato T, Kodaira S, Koyama Y, Sakabe T, Tominaga T, et al. Prognostic factors of colorectal cancer: results of multivariate analysis of curative resection cases with or without adjuvant chemotherapy. Am J Clin Oncol 1996;19:408-15.

16. Huh JW, Oh BR, Kim HR, Kim YJ. Preoperative carcinoembryonic antigen level as an independent prognostic factor in potentially curative colon cancer. J Surg Oncol 2010;101:396-400.

17. Wagener C, Muller-Wallraf R, Nisson S, Groner J, Breuer H. Localization and concentration of carcinoembryonic antigen (CEA) in gastrointestinal tumors: correlation with CEA levels in plasma. J
Natl Cancer Inst 1981;67:539-47.

18. Tabuchi Y, Deguchi H, Saitoh Y. Carcinoembryonic antigen and carbohydrate antigen 19-9 levels of peripheral and draining venous blood in colorectal cancer patients: correlation with histopathologic and immunohistochemical variables. Cancer 1988;62:1605-13.

19. Tabuchi Y, Deguchi H, Imanishi K, Saitoh Y. Comparison of carcinoembryonic antigen levels between portal and peripheral blood in patients with colorectal cancer: correlation with histopathologic variables. Cancer 1987;59:1283-8.

20. Tabuchi Y, Deguchi H, Imanishi K, Saitoh Y. Carcinoembryonic antigen levels of peripheral and draining venous blood in patients with colorectal cancer: correlation with survival. Cancer 1992;69: 2411-7.

21. Thomas P, Zamcheck N. Role of the liver in clearance and excretion of circulating carcinoembryonic antigen (CEA). Dig Dis Sci 1983;28:216-24.

22. Sugarbaker PH. Carcinoembryonic antigen (CEA) assays in obstructive colorectal cancer. Ann Surg 1976;184:752-7. 